\title{
ARTICLE
}

\section{Transcriptomic analysis reveals numerous diverse protein kinases and transcription factors involved in desiccation tolerance in the resurrection plant Myrothamnus flabellifolia}

\author{
Chao Ma ${ }^{1} *$, Hong Wang ${ }^{2 *}$, Andrew J Macnish ${ }^{1}$, Alejandro C Estrada-Melo ${ }^{1}$, Jing Lin ${ }^{2}$, Youhong Chang ${ }^{2}$, Michael S Reid ${ }^{1}$ \\ and Cai-Zhong Jiang ${ }^{3}$
}

The woody resurrection plant Myrothamnus flabellifolia has remarkable tolerance to desiccation. Pyro-sequencing technology permitted us to analyze the transcriptome of $M$. flabellifolia during both dehydration and rehydration. We identified a total of 8287 and 8542 differentially transcribed genes during dehydration and rehydration treatments respectively. Approximately 295 transcription factors (TFs) and 484 protein kinases (PKs) were up- or down-regulated in response to desiccation stress. Among these, the transcript levels of 53 TFs and 91 PKs increased rapidly and peaked early during dehydration. These regulators transduce signal cascades of molecular pathways, including the up-regulation of ABA-dependent and independent drought stress pathways and the activation of protective mechanisms for coping with oxidative damage. Antioxidant systems are up-regulated, and the photosynthetic system is modified to reduce ROS generation. Secondary metabolism may participate in the desiccation tolerance of $M$. flabellifolia as indicated by increases in transcript abundance of genes involved in isopentenyl diphosphate biosynthesis. Up-regulation of genes encoding late embryogenesis abundant proteins and sucrose phosphate synthase is also associated with increased tolerance to desiccation. During rehydration, the transcriptome is also enriched in transcripts of genes encoding TFs and PKs, as well as genes involved in photosynthesis, and protein synthesis. The data reported here contribute comprehensive insights into the molecular mechanisms of desiccation tolerance in $M$. flabellifolia.

Horticulture Research (2015) 2, 15034; doi:10.1038/hortres.2015.34; published online: 22 July 2015.

\section{INTRODUCTION}

Tolerance to desiccation can be defined as the ability of an organism to survive extreme dehydration and then to resume normal growth upon rehydration. ${ }^{1}$ The phenomenon of desiccation tolerance is common in seeds and non-tracheophytes. ${ }^{2}$ Vascular plants with the ability to revive from extreme desiccation are commonly called as resurrection plants. ${ }^{3}$ To date, more than 300 angiosperms, including a few dicotyledonous plants, have been identified as resurrection plants. ${ }^{1,4}$

Tolerance to desiccation in resurrection plants is a complex process that involves many physiological and metabolic mechanisms. ${ }^{1-3}$ The strategies adopted by resurrection plants to survive desiccation may resemble those seen in seeds., 5 ater deficit is initially accompanied by osmotic adjustment to protect against cellular damage, which is accomplished by the accumulation of large amounts of sugars, amino acids, and small polypeptides such as late embryogenesis abundant (LEA) proteins and dehydrins. ${ }^{1}$ Increasing water deficit results in the activation of mechanisms to cope with desiccation-induced structural and functional alterations of macromolecules and membranes, the accumulation of toxic substances and free radicals, and mechanical damage associated with the loss of turgor. ${ }^{1}$ Under desiccation stress, the oxidative damage caused by the production of reactive oxygen species (ROS) is enhanced, especially in chloroplasts. Two different strategies are adopted by resurrection plants to minimize ROS damage. Homoiochlorophyllous resurrection plants conserve the structure of their photosynthetic apparatus, synthesize anthocyanins, and increase the activity of antioxidants during desiccation, whereas poikilochlorophyllous resurrection plants demolish chloroplast pigments and membranes, thus reducing cellular sources of ROS. ${ }^{6}$

Resurrection plants are excellent models for studying molecular mechanisms that could potentially serve as biotechnological tools for enhancing drought tolerance. ${ }^{7}$ There have been many studies of the molecular effects of drought on model plants such as Arabidopsis and rice, ${ }^{8,9}$ but information is scarce on the desiccation tolerance mechanisms of resurrection plants. ${ }^{2,10}$ Transcriptomics has been used to generate global gene expression atlases, such as for powdery mildew resistance, ${ }^{11}$ horticultural traits in apple, ${ }^{12}$ fruit ripening, ${ }^{13}$ and cherry fruit development. ${ }^{14}$ To understand desiccation tolerance mechanisms, transcriptomic and proteomic studies have been performed in resurrection plants such as Sporobolus stapfianus, ${ }^{15}$ Tortula ruralis, $^{16}$ Xerophyta viscosa, ${ }^{17}$ and Boea hygrometrica. ${ }^{18}$ However, large-scale expressed sequence tag sequencing analysis using high-throughput sequencing and de novo assembly strategies have only been conducted in two herbaceous species, Craterostigma plantagineum $^{6,19}$ and Haberlea rhodopensis. ${ }^{20}$

Myrothamnus flabellifolia, a woody homoiochlorophyllous resurrection plant growing in the mountainous regions of central and southern Africa, ${ }^{21}$ is probably the most primitive angiosperm to show extreme tolerance to desiccation. ${ }^{22}$ It displays novel anatomical, ultra-structural, and biochemical adaptations to desiccation stress ${ }^{23}$

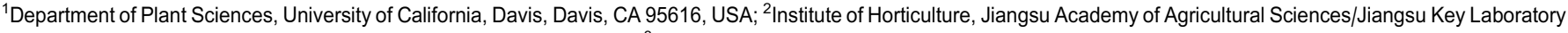
for Horticultural Crop Genetic Improvement, Nanjing 210014, China and ${ }^{3}$ Crops Pathology and Genetic Research Unit, United States Department of Agriculture, Agricultural Research Service, Davis, CA 95616, USA. *These authors contributed equally to this work

Correspondence: Cai-Zhong Jiang (cjiang@ucdavis.edu)

Received: 5 May 2015; revised: 8 June 2015; accepted: 9 June 2015 


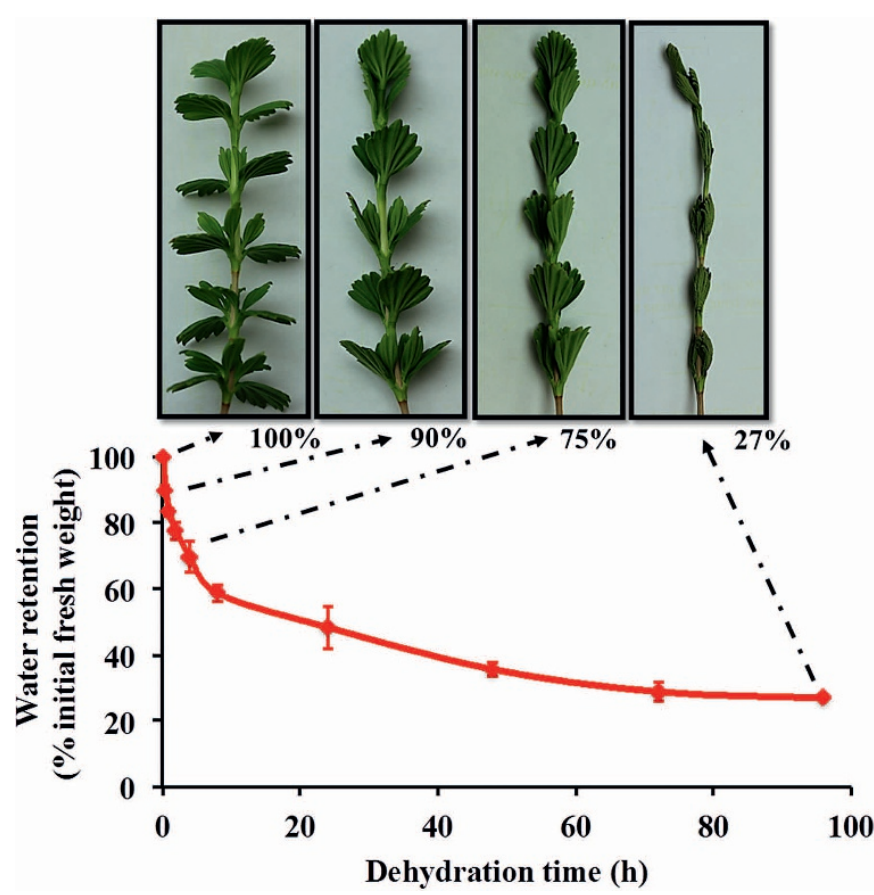

Figure 1. Water loss curve of $M$. flabellifolia. Water loss is expressed as the percentage of IFW at each time point. Results are the means of three biological replicates \pm SD.

and has been the subject of a number of physiological and biochemical studies. Under severe drought conditions, M. flabellifolia leaves can become air-dry, folding in a unique fan-like manner. ${ }^{24}$ Upon rewatering, the leaves return to their original color and shape within 24 hours. ${ }^{25}$ Biochemical studies of the cell walls have demonstrated an abundance of arabinose polymer side chains, consisting of arabinans associated with the pectin matrix, which may explain the flexibility of the mesophyll cells, allowing leaf morphology to be rapidly recovered after rehydration. ${ }^{22,26}$ During desiccation, presumably to protect from ROS, the leaves synthesize large amounts of anthocyanins and change their color from green to dull-brown. ${ }^{21,23}$ High levels of phenols, including tannins, arbutin, and 3,4,5 tri-O-galloylquinic acid, have been reported in the leaves of $M$. flabellifolia. ${ }^{27-29}$ Differences in phenolic content and composition may be associated with differences in tolerance to desiccation stress among populations of M. flabellifolia. ${ }^{30}$ Moreover, desiccated leaves of M. flabellifolia contain high levels of saccharides, although the precise constituents differ. ${ }^{29,31,32}$ The molecular mechanisms underlying the tolerance of $M$. flabellifolia and its ability to rapidly rehydrate are still largely unknown. In the present work, we attempt to investigate the transcriptome dynamics in response to dehydration and rehydration in M. flabellifolia. Most woody fruit and ornamental plants can encounter extreme drought stress. Therefore, understanding this plant's extreme tolerance to desiccation and drought can aid the development of strategies for improving drought stress resistance in horticultural crops. ${ }^{33,34}$

\section{MATERIALS AND METHODS}

Plant materials and dehydration treatment

M. flabellifolia plants were kindly provided by Dr. Matthew Opel (University of Connecticut) and grown in containers in the greenhouse at the University of California, Davis under a $16 \mathrm{~h} / 8 \mathrm{~h}$ day/night cycle. Current season branches from two-year-old plants were used for the treatments. For the dehydration treatment, fully hydrated branches were harvested and placed in a high humidity chamber for 30 min to reduce any potential initial wounding effects. Dehydration was performed by placing the cut branches in a temperature-controlled $\left(20^{\circ} \mathrm{C}\right)$ room at approximately $40 \%$ relative humidity. Mature leaves were collected when the branches weighed $90 \%$ (onset of leaf wilting), $75 \%$ (leaf wilted), or $27 \%$ (desiccated) of their initial fresh weight (IFW) (Figure 1). For rehydration, desiccated branches were submerged in water for $6 \mathrm{~h}$ (onset of leaf unfolding) and $12 \mathrm{~h}$ (leaves recovered). Mature leaves from all these stages were collected and immediately frozen in liquid

\section{Up-regulated genes}

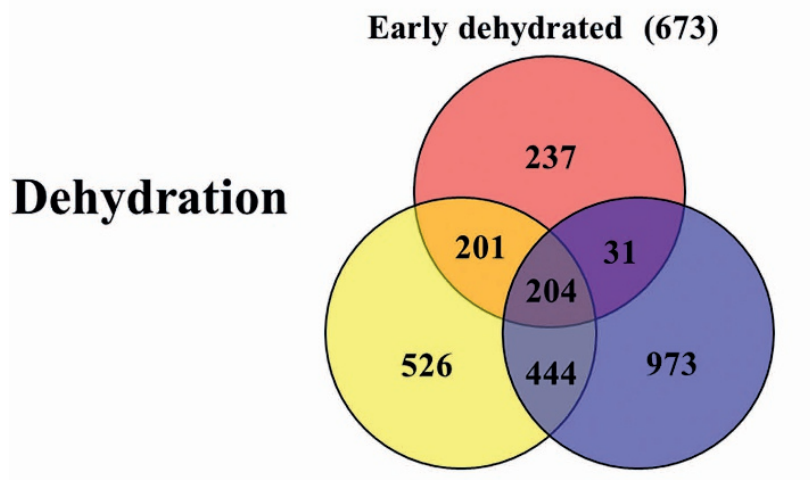

Moderately dehydrated (1375) Desiccated (1652)

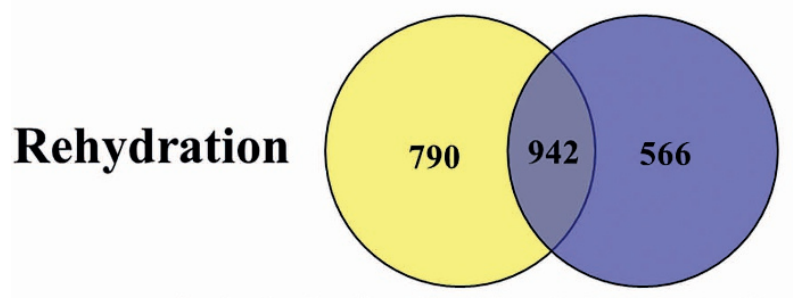

6-h Rehydration (1732)

2-h Rehydration (1508)

\section{Down-regulated genes}

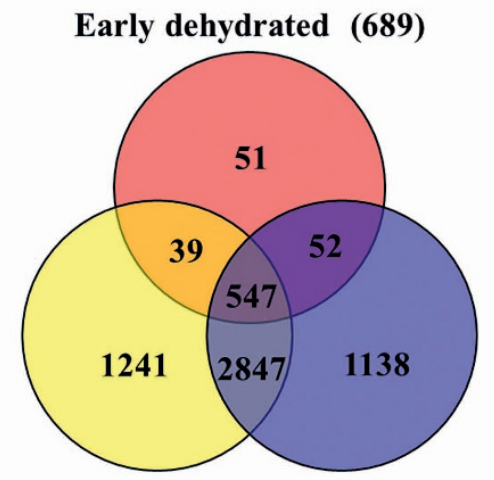

Moderately dehydrated (4674) Desiccated (4584)

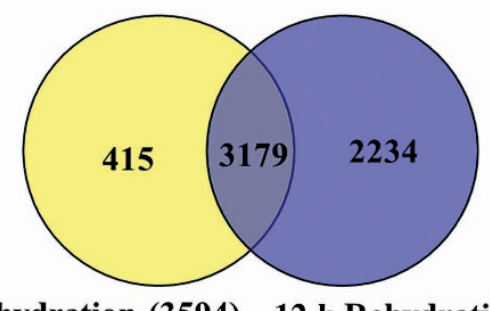

6-h Rehydration (3594) 12-h Rehydration (5413)

Figure 2. Venn diagrams showing common genes significantly up- or down-regulated in dehydration and rehydration processes. 
Table 1. Summary of M. flabellifolia sequencing data

\begin{tabular}{lrrrrrrr}
\hline & \multicolumn{1}{c}{ Control } & Early dehydration & Moderate dehydration & Desiccation & 6-h rehydration & 12-h rehydration & Total \\
\hline No. of reads & 20329227 & 23606535 & 22923710 & 23274062 & 23792776 & 24482833 & 138409143 \\
No. of contigs & 23686 & 23625 & 22895 & 22619 & 22751 & 22837 & 25235 \\
Average contig length (bp) & 2024 & 2034 & 2062 & 2070 & 2069 & 2050 & 1988 \\
\hline
\end{tabular}

nitrogen and stored at $-80^{\circ} \mathrm{C}$ until use. Three biological replicates were mixed for the preparation of an RNA-Seq library.

Total RNA extraction and RNA-Seq library preparation

Total RNA was extracted using the Spectrum Plant Total RNA Kit (SigmaAldrich, St. Louis, MO, USA) and treated with RNase-free DNase I (Promega, Madison, WI, USA) to remove any contaminating genomic DNA. RNA libraries
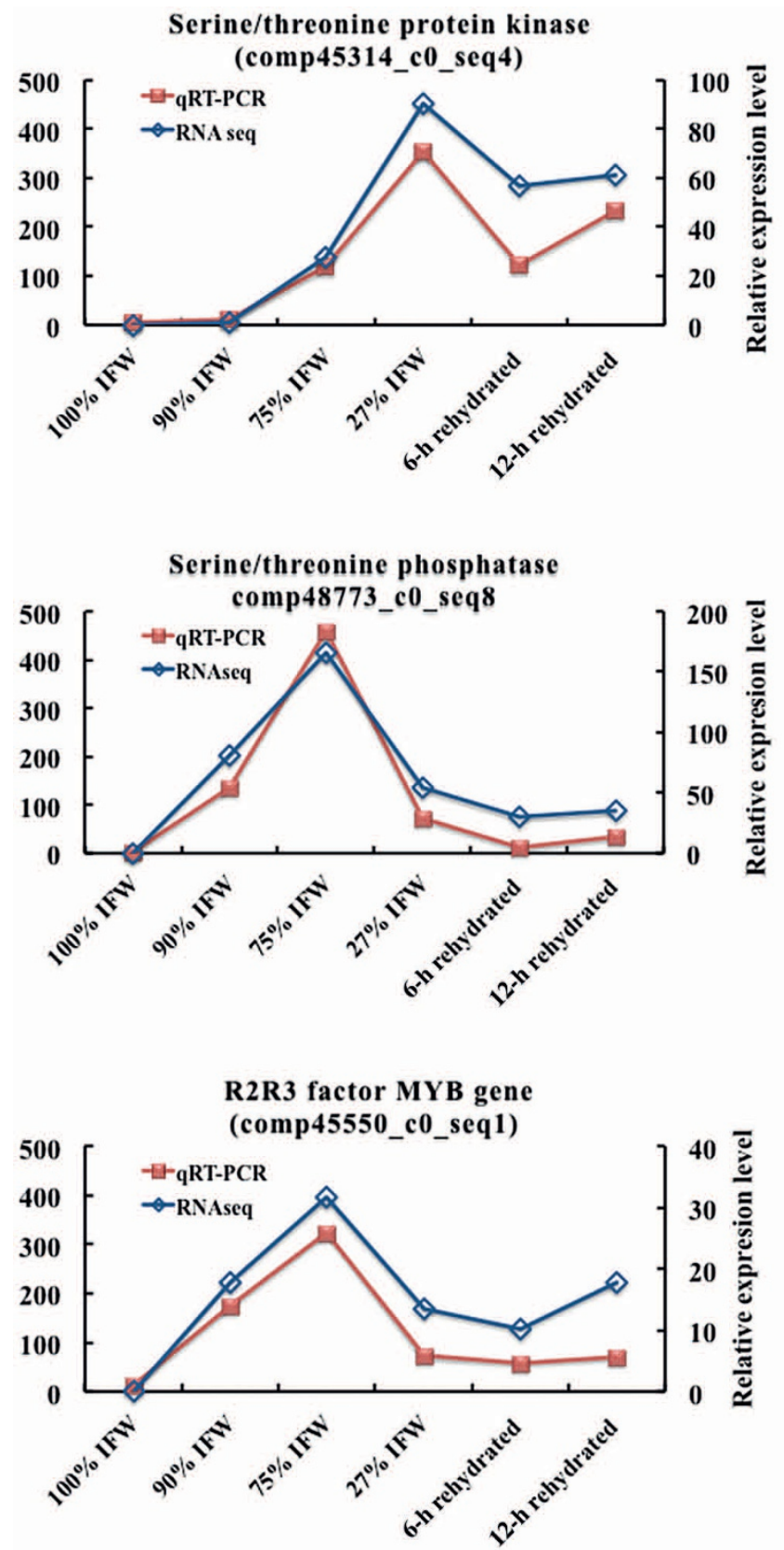

were constructed according to the manufacturer's instructions and sequenced on a HiSeq2000 Illumina platform.

Cleaning and assembly of RNA-Seq sequenced reads

The quality of the raw reads was examined before and after trimming using FastQC software (http://www.bioinformatics.babraham.ac.uk/projects/ fastqc/). Low-quality reads with a Phred quality score less than 20 , sequences

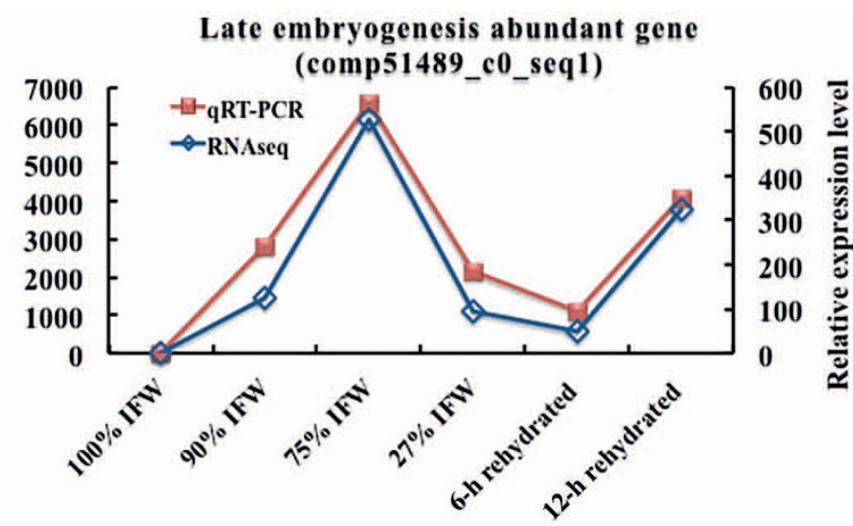

Protein phosphatase $2 \mathrm{~A}$
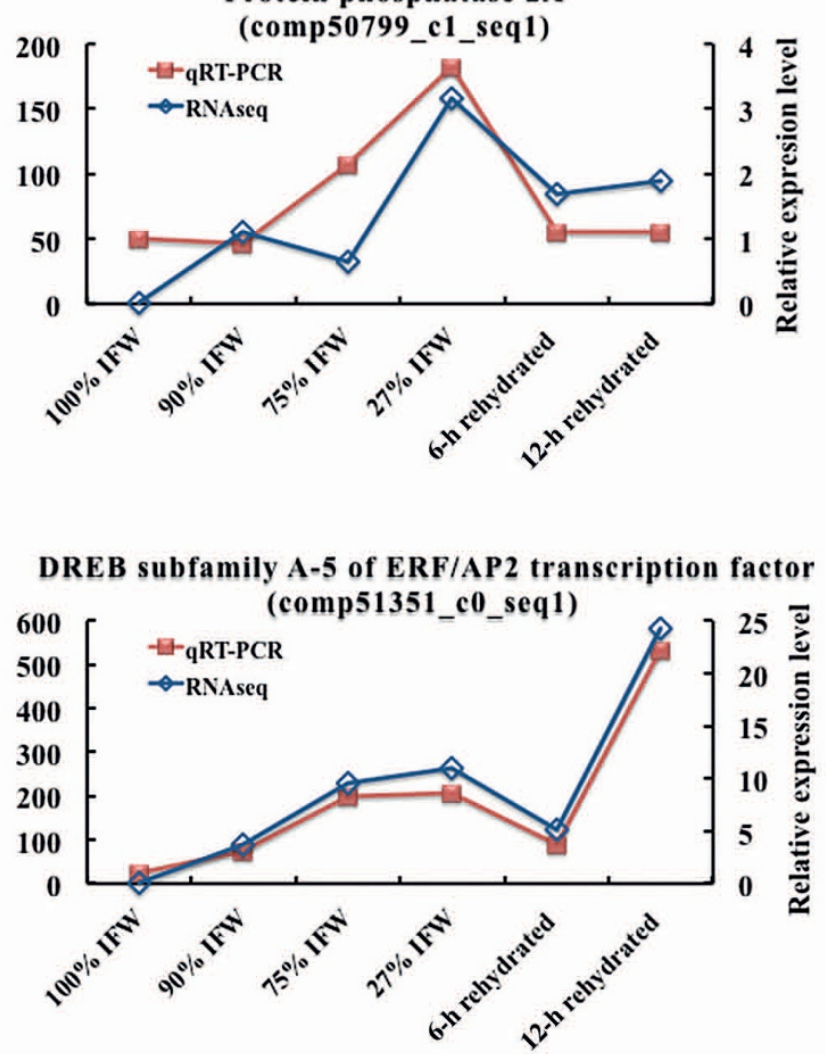

Figure 3. Validation of RNA-Seq data by qRT-PCR. RNA was extracted from leaf tissue taken at the indicated percentage of IFW under dehydration and at the indicated times of rehydration. Actin (comp22823_c0_seq1) was used as an internal control. The results are the means of three biological replicates \pm SD. 


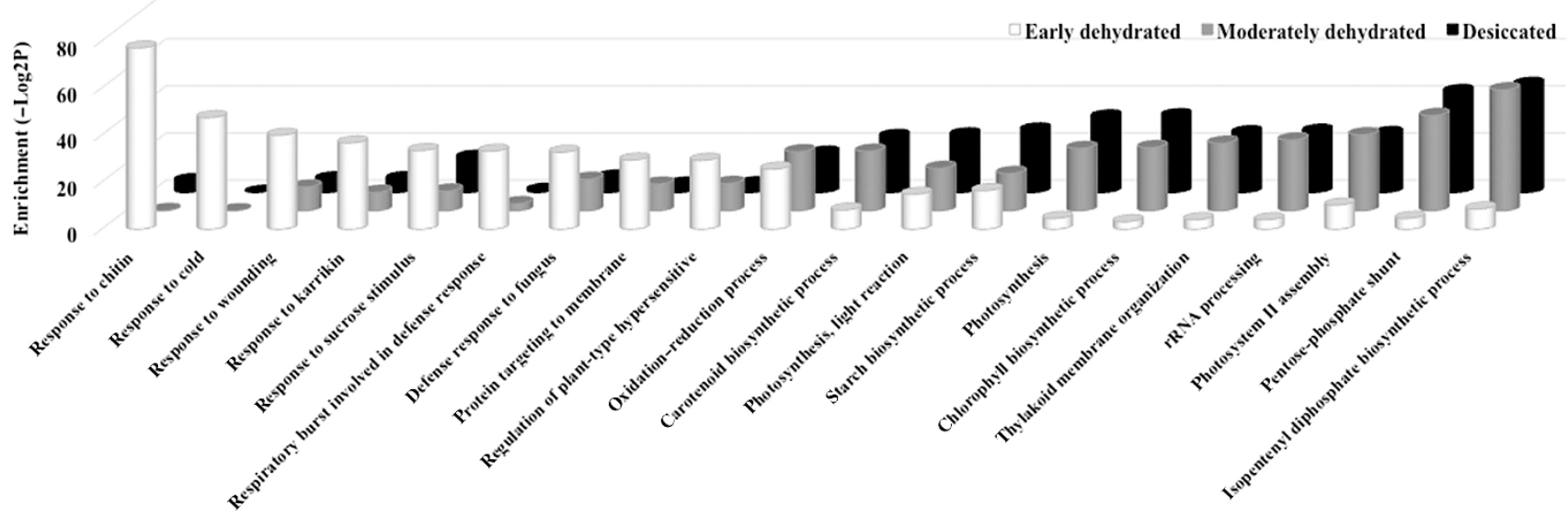

b

GO biological process class

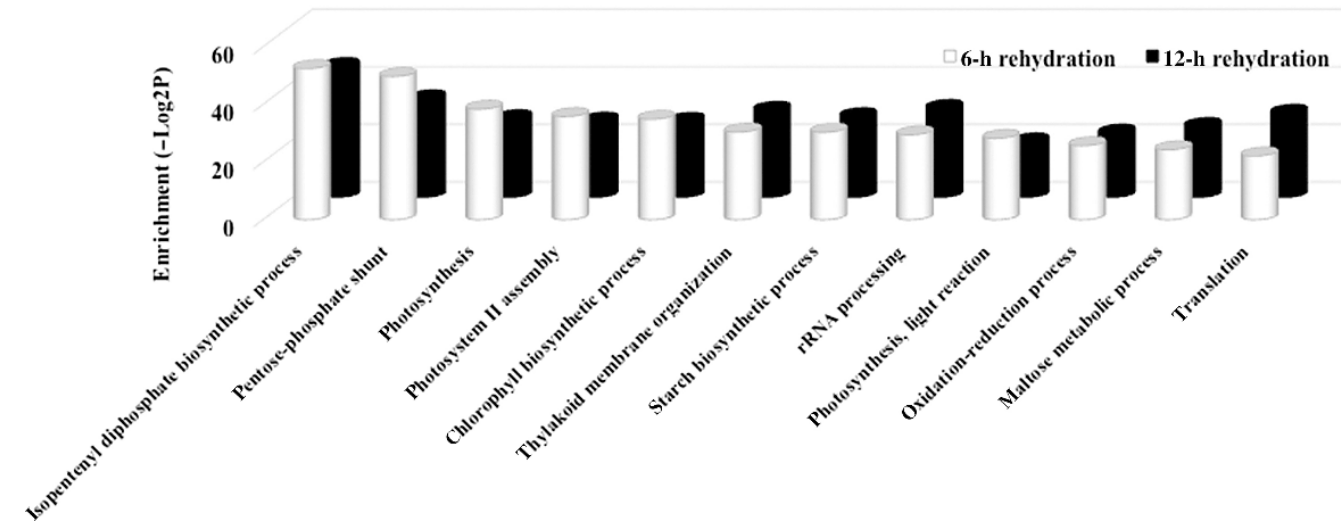

Figure 4. GO functional classification analysis of DTGs during dehydration (a) and rehydration (b). Histograms representing enriched functional distributions in each stage.

shorter than $40 \mathrm{bp}$, barcodes, polyA, polyT ends and adapter sequences were removed. High-quality clean reads were assembled de novo into contigs using the Trinity program. ${ }^{35}$ The resulting contigs were blasted against the PlantGDB and Arabidopsis protein databases with a cutoff E-value of $1 e^{-6}$.

\section{Quantitative real-time PCR analysis}

To examine the transcript abundance of selected genes, total RNA was extracted from leaves of three replicate branches. First strand cDNA was synthesized using $2 \mu \mathrm{g}$ total RNA, oligo (dT) primers, random hexamers, and Superscriptlll reverse transcriptase (Invitrogen, Carlsbad, CA, USA). Quantitative real-time PCR (qRT-PCR) was performed with the 7300 Real Time PCR System (Applied Biosystems, Foster City, CA, USA) using SYBR Green Real-time PCR Master Mix (Applied Biosystems). ${ }^{36}$ To normalize sample variance, actin (comp22823 c0 seq1) was used as an internal control. ${ }^{37}$ Relative quantification of the expression of each gene was performed using the $2^{-\Delta \Delta C T}$ method. ${ }^{38}$ The primers used for determining transcript abundance are listed in Supplementary File 3.

\section{Annotation and pathway enrichment}

Gene ontology (GO) terms were assigned to the assembled contigs based on their homologues in the Arabidopsis protein database. GO term enrichment was performed by a hypergeometric distribution test with Bonferroni multiple testing correction. To investigate metabolic pathways, we compared the contigs with their orthologs in the Arabidopsis KEGG (http://www.genome.jp/dbget/). Transcription factors (TFs) were identified and classified as such if their Arabidopsis homologs were identified in the Arabidopsis TF database (http://arabidopsis.med.ohio-state.edu/AtTFDB/). Protein kinases (PKs) were identified and classified as such if their Arabidopsis homologs were identified in the list of PKs in the PlantsP database (http://plantsp.genomics.purdue.edu/index.html).

Hierarchical clustering

GenePattern (http://genepattern.broadinstitute.org/gp/pages/index.jsf) was used for hierarchical clustering. Default options were used and the Pearson correlation was performed on gene expression data.

\section{RESULTS AND DISCUSSION}

Sequencing and transcriptome assembly of $M$. flabellifolia Six RNA-Seq libraries of $M$. flabellifolia were constructed from leaves of branches that were fully hydrated (control), slightly dehydrated (90\% IFW), moderately dehydrated (75\% IFW), desiccated (27\% IFW), and rehydrated for $6 \mathrm{~h}$ or $12 \mathrm{~h}$ (Figure 1). A total of 141262112 paired-end raw reads of 100 nucleotides (nt) were generated using the Illumina platform. These raw sequences were further filtered and cleaned up, resulting in 138409143 paired-end clean reads (Table 1). The proportion of clean reads to total raw reads was over $98 \%$ in each library. These high-quality reads generated 25235 contigs by de novo assembly, with an average contig length of $1988 \mathrm{nt}$ (Table 1). We annotated the assembled contigs through homologous searching (BlastX) against the PlantGDB database. A total of 14273 (56.6\%) contigs had at least one homolog in the PlantGDB database. BlastX analysis against PlantGDB revealed that translated proteins from many of $M$. flabellifolia's transcripts were similar to the protein 

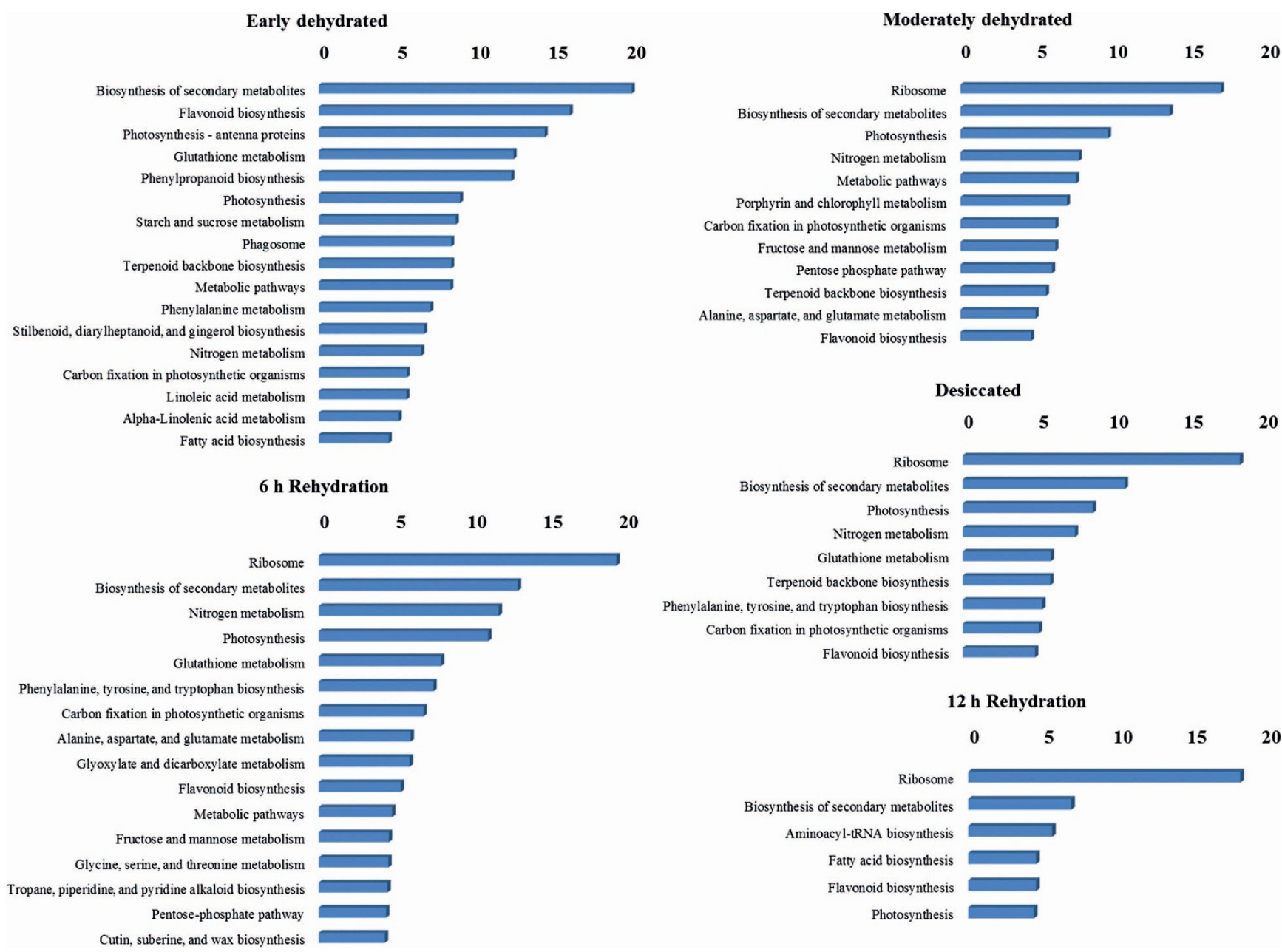

20

Carbon fixation in photosynthetic organism

Flavonoid biosynthesis

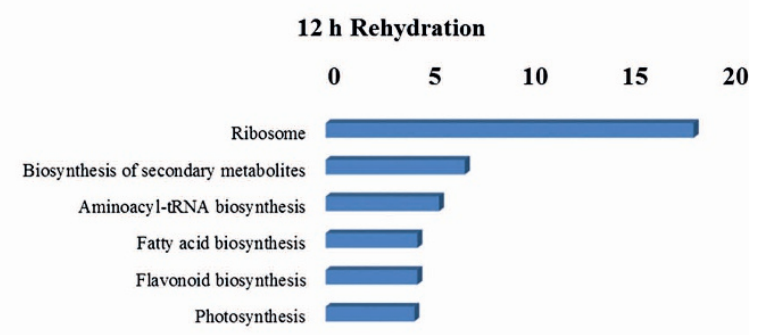

Figure 5. Enriched pathways of DTGs during dehydration and rehydration.

sequences in Prunus persica (30.4\%), Manihot esculenta (28.5\%), and Populus trichocarpa (24.9\%) (Supplementary File 1).

Changes in transcriptome profiles during dehydration and rehydration

A total of 8287 differentially transcribed genes (DTGs) were detected in the dehydration treatments (Supplementary File 4). The overlapping regions of the Venn diagram in Figure 2 show that 204 DTGs were up-regulated and 547 DTGs were down-regulated during all the dehydration stages. The number of up- and downregulated DTGs increased as desiccation progressed (Figure 2), indicating increasing molecular responses as dehydration stress increased. During rehydration, a total of 8542 DTGs were identified (Supplementary File 5). Among these DTGs, 942 up-regulated DTGs and 3179 down-regulated DTGs were present in both the $6 \mathrm{~h}$ and 12 $\mathrm{h}$ rehydration stages (Figure 2 ).

To validate the DTGs identified by RNA-Seq, we analyzed the transcript abundance of six randomly selected DTGs using qRTPCR analysis. The results obtained from qRT-PCR analysis were generally consistent with the expression analysis generated from the RNA-Seq data (Figure 3).

Functional assignment of differentially transcribed genes during dehydration and rehydration

To evaluate the potential functions of genes that showed significant transcriptional changes during dehydration and rehydration in M. flabellifolia, we examined the GO enrichment of DTGs at each dehydration and rehydration stage. The biological processes that were significantly enriched early in dehydration included many defense responses such as response to chitin, cold, wounding, and fungi (Figure 4a). This is consistent with a previous transcriptomic study in the resurrection plant $H$. rhodopensis in which many genes involved in acquiring tolerance to a variety of abiotic and biotic stresses were induced by desiccation stress. ${ }^{20}$ In striking contrast, the DTGs represented in moderately dehydrated and desiccated leaves participate in photosystem II assembly, thylakoid membrane organization, chlorophyll biosynthesis, and photosynthesis (Figure 4a). These data suggest that alteration of photosynthesis is an important strategy in response to desiccation for M. flabellifolia. Moreover, genes involved in isopentenyl diphosphate biosynthesis were enriched in moderately dehydrated, desiccated, and rehydrated leaves (Figure 4a and b). Isopentenyl disphosphate is a precursor of all plant isoprenoids, ${ }^{39}$ which are a diverse group of metabolites including primary metabolites such as sterols, chlorophylls, carotenoids, quinones, and hormones (brassinosteroids, cytokinins, gibberellins, abscisic acid ( $A B A)$ ) and secondary metabolites participating in plant defense and communication such as pigments, volatiles, and defense compounds. ${ }^{40,41}$

To further delineate the metabolic pathways participating in the dehydration and rehydration responses, we mapped the DTGs into the Kyoto Encyclopedia of Genes and Genomes (KEGG) database. A total of 23 and 18 biochemical pathways were significantly enriched during dehydration and rehydration, respectively (Figure 5). During dehydration, consistent with the GO enrichment 

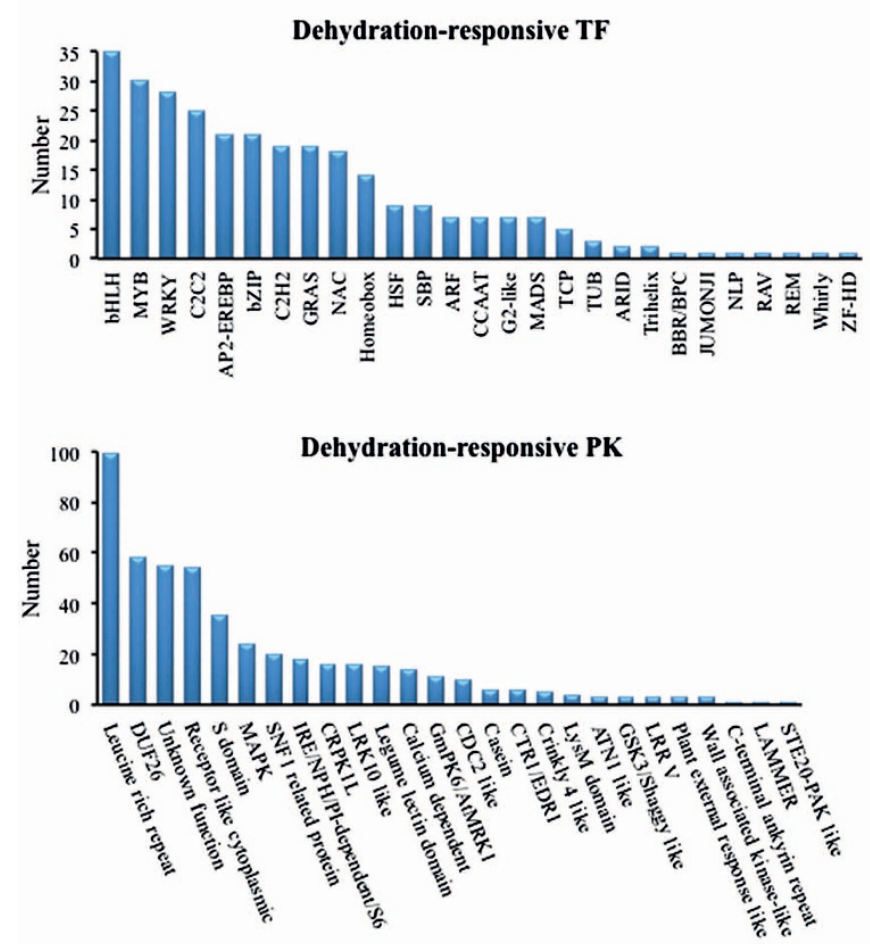
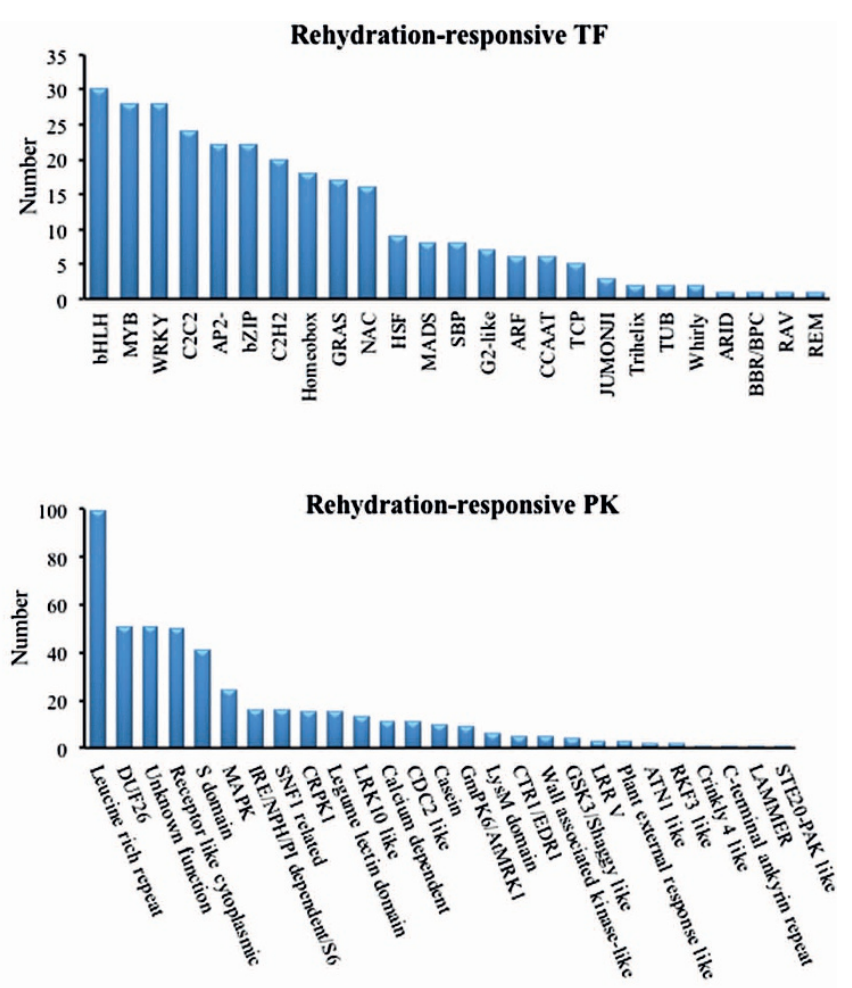

Figure 6. Distribution of dehydration- and rehydration-responsive transcription factors and protein kinases in M. flabellifolia.

analysis, the enriched biochemical pathways involved diverse metabolites, including secondary metabolites, starch, sucrose, fatty acids, fructose and mannose, and photosynthesis, suggesting a global response to desiccation in M. flabellifolia. As one example, the flavonoid biosynthesis pathway was significantly enriched during dehydration, consistent with the role of anthocyanins, a flavonoid class, in the 'green-to-brown'/'brown-to-green' leaf color alternation during dehydration and rehydration. ${ }^{23}$

During rehydration, photosynthetic genes were significantly enriched (Figure 5). This is consistent with a previous study in M. flabellifolia showing that photosynthesis resumes rapidly, reaching full capacity within three days of rehydration. ${ }^{42}$ The recovery of vital functions and metabolic activities during rehydration is indicated by alterations of genes encoding a range of structural and physiological processes. ${ }^{1}$ For example, genes encoding enzymes involved in ribosome and aminoacyl-rRNA biosynthesis, essential for the re-establishment of normal cellular metabolism, are enriched during rehydration (Figure 5).

Dehydration- and rehydration-responsive transcription factors and protein kinases in M. flabellifolia

Transcription factors (TFs) act as master switches of transcriptional reprogramming, inducing diverse protective mechanisms in response to abiotic stresses. $9,43,44$ We identified a large number of TFs (295) responsive to dehydration, and 287 TFs were responsive to rehydration from among the DTGs (Supplementary Files 2 and 6). Among these TFs, the $b H L H, M Y B$, and WRKY families were the largest groups during both dehydration and rehydration (Figure 6). Members of these three TF families are implicated in stress responses in model plants. In Arabidopsis, bHLH-type genes AtMYC2 and AtAIB are involved in ABA signaling, and overexpression of $A t M Y C 2$ or $A t A I B$ enhances the drought tolerance of transgenic plants. ${ }^{45,46}$ Multiple $M Y B$ genes have also been implicated in stress responses. For example, AtMYB2 is involved in the ABA-dependent drought tolerance pathway, ${ }^{45}$ and AtMYB108 is associated with both biotic and abiotic stress responses. ${ }^{47}$ WRKY
TFs are well known for their involvement in the regulation of plant development and in response to abiotic stresses. ${ }^{48,49}$ Functional analyses of rice OsWRKY11 and soybean GmWRKY54 demonstrated that WRKYs are involved in drought signaling pathways. ${ }^{50}$ SBP (SQUAMOSA promoter binding protein) and TCP TFs are known to be plant-specific developmental regulators. ${ }^{51}$ Our results revealed that 9 SBP and 5 TCP type TFs also showed a response to dehydration in M. flabellifolia (Figure 6 and Supplementary File 6). Further functional characterization of these TFs may shed light on their roles in desiccation tolerance.

PKs are essential signaling regulators in the acquisition of desiccation tolerance. Among the DTGs, we identified strikingly large numbers that respond to dehydration (484 PKs) and rehydration (468 PKs) (Supplementary Files 2 and 7). PKs may play a key regulatory role in drought stress adaptation in $M$. flabellifolia, as corroborated by comparison of our data with Arabidopsis ${ }^{52}$ and chrysanthemum drought stress expression profile data. ${ }^{53}$ In Arabidopsis, only 121 PKs were found to be responsive to osmotic stress. ${ }^{52}$ Xu et al. (2013) identified only 229 differentially expressed PKs during drought stress in chrysanthemum, a species with a large and complex polyploid genome and high heterozygosity. ${ }^{54}$

Protein family distribution analyses showed that three receptorlike kinase (RLK) type families - leucine-rich repeat (LRR) kinases, domain of unknown function DUF26, and receptor like cytoplasmic kinases - were the largest groups of DTGs in both the dehydration and rehydration treatments (Figure 6). RLKs constitute the largest PK family in plants ${ }^{55}$ and have been implicated in the regulation of meristem proliferation, organ specification, reproduction, and hormone signal transduction. ${ }^{55-57}$ Drought transcript profiling in Arabidopsis has revealed that the transcript abundance of many RLK genes peaks $1 \mathrm{~h}$ after the start of drought treatment, indicating that RLKs may also be part of a rapid drought response. ${ }^{58}$ Several functional studies have also confirmed roles of RLKs in drought tolerance. ${ }^{56}$ In Arabidopsis, an LRR kinase, receptor protein kinase 1 (RPK1), is induced by ABA. Repression of RPK1 decreases sensitiv- 
a
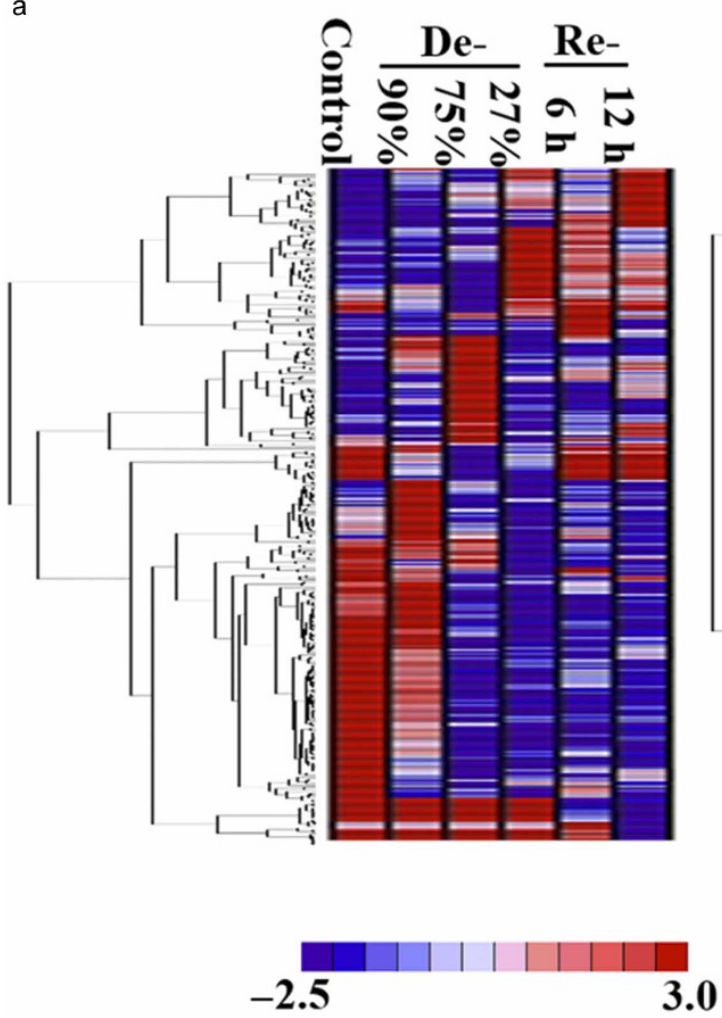

b

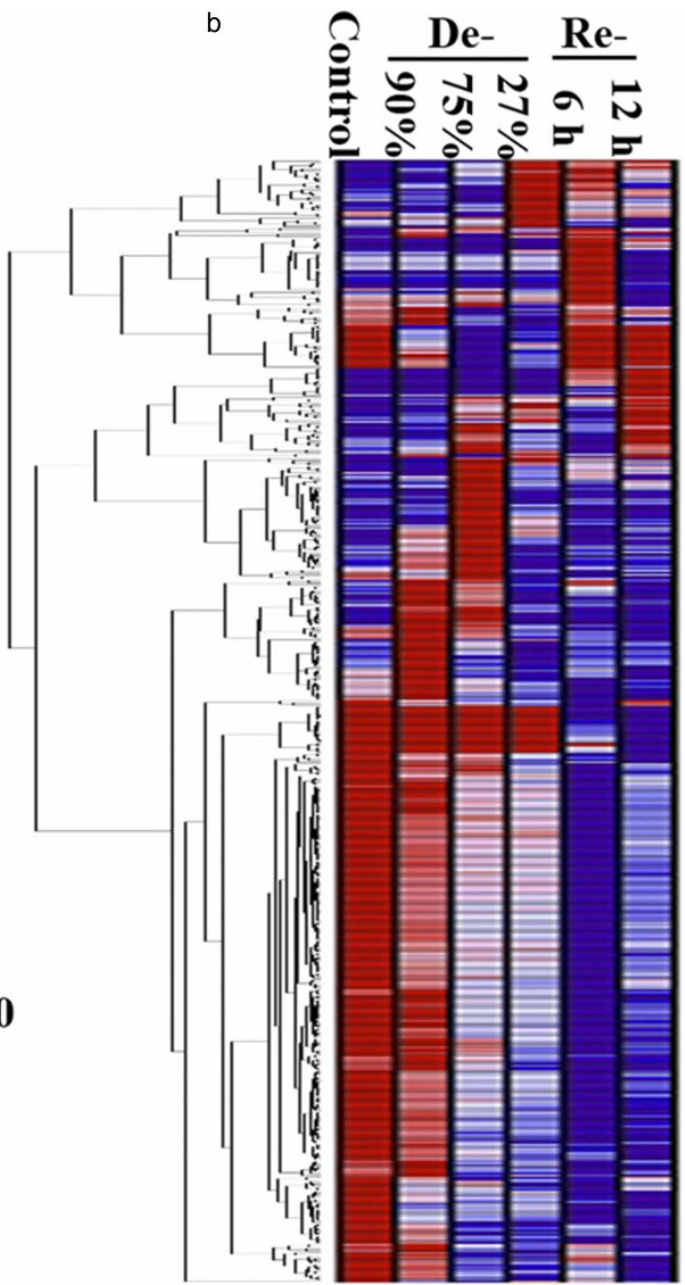

Figure 7. Hierarchical cluster analysis of differentially expressed transcription factors (a) and protein kinases (b) in M. flabellifolia. Log2 ratios are used to express the relative transcript abundance at the indicated percentage of IFW during dehydration (De-) or at the indicated time of rehydration (Re-) of leaves versus control hydrated leaves. Red squares indicate increased expression and blue squares indicate decreased.

ity to $A B A$, suggesting that RPK1 is involved in ABA perception. ${ }^{59}$ A DUF26 type kinase, CRK36, and a receptor-like cytoplasmic type kinase, ARCK1, form a complex that negatively controls ABA and osmotic stress signal transduction. ${ }^{60}$

In addition, 24 DTGs encoding mitogen-activated protein kinases (MAPKs) were identified during dehydration stress in M. flabellifolia (Figure 6 and Supplementary File 7). MAPK cascades function in transducing environmental and developmental cues to intracellular responses. ${ }^{61}$ In Arabidopsis, several MAPKs are involved in abiotic stress signaling pathways including MEKK1, ${ }^{62}$ MPK1, ${ }^{63}$ MPK3, ${ }^{62,64,65}$ MPK4, ${ }^{63}$ MPK6, ${ }^{63}$ MAP9, ${ }^{66}$ and MAP12. ${ }^{66}$ The transcript abundance of homologs of all these MAPKs, apart from MPK6 and MAP12, was altered in response to dehydration in $M$. flabellifolia (Supplementary File 7).

To identify early responsive regulatory genes, the expression pattern of differentially transcribed TFs and PKs was analyzed by hierarchical clustering (Figure 7). The transcript abundance of 53 TFs and 91 PKs peaked early in dehydration (Figure 8, Supplementary Files 8 and 9), indicating that broad regulatory networks are quickly employed to coordinate global transcriptional reprogramming during desiccation of $M$. flabellifolia. Among the up-regulated TFs during early dehydration, the strongest induction was observed for genes encoding putative DREB family members (comp45691_c0_seq1, comp42806_c0_seq1, Supplementary File 8).
The DREB TFs are well-known regulators of ABA-independent drought stress signaling pathways in other plant species. ${ }^{9,46,67}$ In addition, a total of nine genes encoding WRKY family members were included among the up-regulated TFs in early dehydration, making the WRKY family the biggest group of early dehydration up-regulated TFs (Supplementary File 8). Furthermore, among the up-regulated PKs, the LRK10 L kinase family is one of the largest groups in during early dehydration (13 out of total 91 PKs) (Supplementary File 9), suggesting that LRK10 L kinases may be important upstream regulators of rapid responses to water deficit. For example, the transcript abundance of a DTG (comp49162_c0_seq1) encoding an LRK10 L kinase increased nearly 120 -fold in early dehydration (Supplementary File 9). LRK10 L genes have previously been reported to be associated with resistance to pathogen infection. ${ }^{68-70}$ Functional characterization of these LRK10 L kinases will be an important step toward elucidating their roles in desiccation tolerance.

Induction of diverse mechanisms in response to desiccation stress in M. flabellifolia

$A B A$ signaling. The plant hormone $A B A$ plays a critical role during dehydration stress. ${ }^{9,43}$ Evidence from earlier studies showed that $A B A$ is also important for desiccation tolerance in resurrection plants such as C. plantagineum ${ }^{71}$ and Chamaegigas intrepidus. ${ }^{72}$ ABA con- 


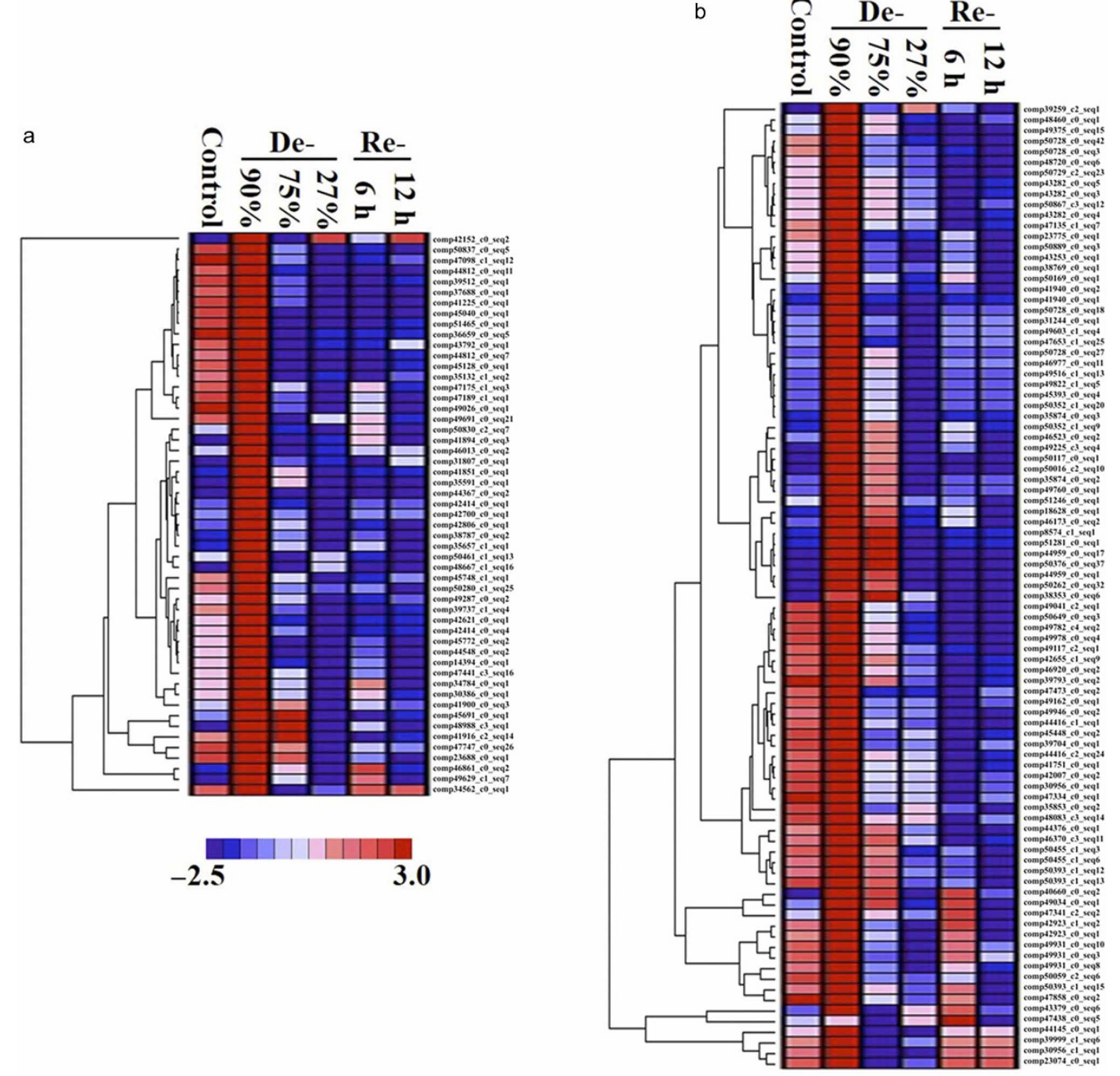

Figure 8. Hierarchical clustering analysis of early dehydration up-regulated transcription factors (a) and protein kinases (b) in M. flabellifolia. Log2 ratios are used to express relative transcript abundance at the indicated percentage of IFW under dehydration (De-) or at the indicated time of rehydration (Re-) of leaves versus hydrated leaves. Red squares indicate increased abundance and blue squares indicate decreased abundance.

tent increases in desiccated leaves of M. flabellifolia, and desiccation stress tolerance is enhanced by exogenous ABA treatment in $M$. flabellifolia. ${ }^{73}$ In agreement with the physiological studies, our desiccation transcriptomic results showed that many ABA biosynthesis and signaling genes were up-regulated during desiccation (Figure 9 and Supplementary File 10). For example, transcripts of four homologs of the nine-cis-epoxycarotenoid dioxygenase (NCED) gene (comp41624_c0_seq1, comp47700_c0_seq4, comp49970_c0_seq2, comp40276_c0_seq1, Figure 9, Supplementary File 10), encoding the rate limiting enzyme in ABA biosynthesis, were increased during dehydration. Similarly, transcripts of several $A B A$ signaling-related genes were also up-regulated, including ABF2 (comp42333_c0_ seq1), $A B 11$ (comp47878_c0_seq10), and HAI2 (comp48773_c0_seq8) (Figure 9 and Supplementary File 10).

Late embryogenesis abundant proteins. Many LEA proteins are synthesized when plants respond to desiccation, suggesting that these proteins may play an important role in desiccation tolerance. ${ }^{43,74}$ In the resurrection plants C. plantagineum and $H$. rhodopensis, large-scale analysis of desiccation-tolerant transcrip- tome data showed that up to nine LEA genes are highly accumulated in desiccated leaves. ${ }^{6,20}$ In M. flabellifolia, transcripts of 18 DTGs encoding LEA proteins were highly accumulated and remained at relatively high levels throughout dehydration and rehydration (Table 2). Intriguingly, compared with those LEAs in C. plantagineum ${ }^{6}$ and $H$. rhodopensis, ${ }^{20}$ we found that highly related LEA orthologs were all up-regulated under desiccation stress among these three resurrection plants (Table 2), suggesting that specific LEA genes are employed to enhance desiccation stress tolerance in resurrection plants.

Photosynthesis. M. flabellifolia, as a homoiochlorophyllous resurrection plant, maintains its photosynthetic apparatus and chlorophyll under desiccation stress. ${ }^{21}$ The advantage of this is the ability to rapidly resume photosynthesis during rehydration. However, retaining the photosynthetic apparatus during desiccation exposes plants to the risk of oxidative damage caused by the extra production of ROS by photosynthetic pigments, photosystems and cofactors. We found that the transcript abundance of several genes encoding antioxidants was increased by dehydration treatment 

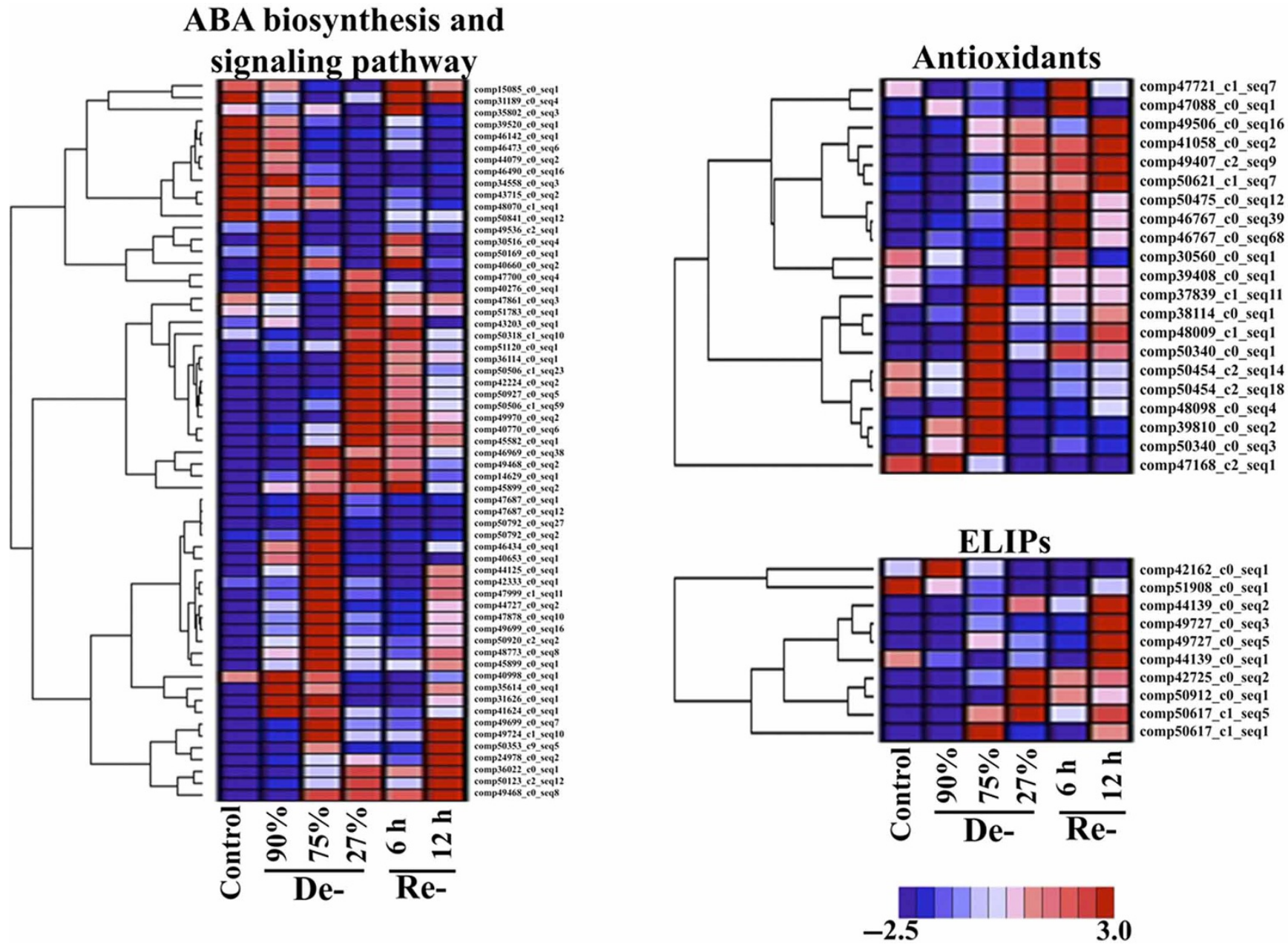

Figure 9. Representative pathways in de- and re-hydration treatments in M. flabellifolia transcript profiles. Log2 ratios are used to express relative transcript abundance at the indicated percentage of IFW during dehydration (De-) or at the indicated time of rehydration (Re-) of leaves versus hydrated leaves. Red squares indicate increased abundance and blue squares indicate decreased abundance.

Table 2. Expression profiles of LEA genes in M. flabellifolia

\begin{tabular}{|c|c|c|c|c|c|c|c|c|}
\hline ContigID & Control & $\begin{array}{c}\text { Early } \\
\text { dehydration }\end{array}$ & $\begin{array}{c}\text { Moderate } \\
\text { dehydration }\end{array}$ & Desiccation & 6-h rehydration & $\begin{array}{l}12-\mathrm{h} \\
\text { rehydration }\end{array}$ & Putative ortholog & Other plants ${ }^{a}$ \\
\hline Comp40530_c0_seq1 & 2.25 & 268.53 & 556.06 & 67.76 & 28.93 & 301.87 & At1 g72100 & c \\
\hline Comp50751_c0_seq1 & 7.69 & 787.21 & 3792.9 & 735.86 & 300.26 & 1945.18 & At2 g21490 & \\
\hline Comp42145_c0_seq1 & 16.39 & 2159.51 & 9526.27 & 1810.41 & 841.27 & 6780.48 & At2 g36640 & $\mathrm{H}, \mathrm{C}$ \\
\hline Comp42145_c0_seq4 & 4.99 & 1117.04 & 4653.76 & 869.67 & 460.52 & 3248.48 & At2 g36640 & $\mathrm{H}, \mathrm{C}$ \\
\hline Comp46243_c0_seq2 & 1.11 & 17.86 & 159.84 & 122.67 & 70.21 & 626.39 & At2 g36640 & $\mathrm{H}, \mathrm{C}$ \\
\hline Comp46692_c0_seq4 & 1.03 & 14.69 & 169.17 & 135.22 & 83.26 & 635.56 & At2 g42560 & \\
\hline Comp43396_c2_seq3 & 17.49 & 1741.06 & 11861.78 & 3305.72 & 1783.88 & 13218.9 & At3 g15670 & $\mathrm{H}, \mathrm{C}$ \\
\hline Comp43396_c2_seq4 & 12.52 & 1475.64 & 5200.8 & 261.22 & 154.19 & 1116.84 & At3 g15670 & $\mathrm{H}, \mathrm{C}$ \\
\hline Comp51407_c0_seq1 & 70.14 & 432.55 & 794.2 & 4.53 & 0.5 & 234.9 & At3 g15670 & $\mathrm{H}, \mathrm{C}$ \\
\hline Comp50084_c1_seq1 & 11.88 & 550.75 & 3750.64 & 760.76 & 547.69 & 1917.12 & At3 g17520 & \\
\hline Comp39347_c0_seq2 & 29.03 & 36.63 & 85.01 & 10.08 & 7.06 & 1.55 & At3 g53040 & C \\
\hline Comp51118_c0_seq1 & 45.46 & 794.72 & 1886.8 & 76.77 & 29.26 & 381 & At3 g53040 & C \\
\hline Comp51489_c0_seq1 & 9.58 & 1480.88 & 6149.5 & 1117.44 & 577.98 & 3797.75 & At3 g53040 & C \\
\hline Comp52111_c0_seq1 & 7.92 & 797.48 & 3334.65 & 625.14 & 309.71 & 2052.38 & At3 g53040 & C \\
\hline Comp46226_c0_seq1 & 3.44 & 125.3 & 881.84 & 88.37 & 71.15 & 208.33 & At4 g36600 & \\
\hline Comp49017_c4_seq10 & 17.51 & 146.51 & 474.57 & 43.51 & 21.4 & 128.54 & At5 g06760 & c \\
\hline Comp45763_c0_seq1 & 25.1 & 1142.38 & 3559.46 & 1834.16 & 1579.35 & 4230.23 & At5 g44310 & C \\
\hline
\end{tabular}

The expression level values are the normalized read counts in the dehydration and rehydration processes.

${ }^{\text {aT }}$ These $L E A$ genes have been previously reported to be up-regulated during desiccation stress in the listed resurrection plant species.

$\mathrm{H}$, Haberlea rhodopensis; ${ }^{20} \mathrm{C}$, Craterostigma plantagineum. ${ }^{6}$ 


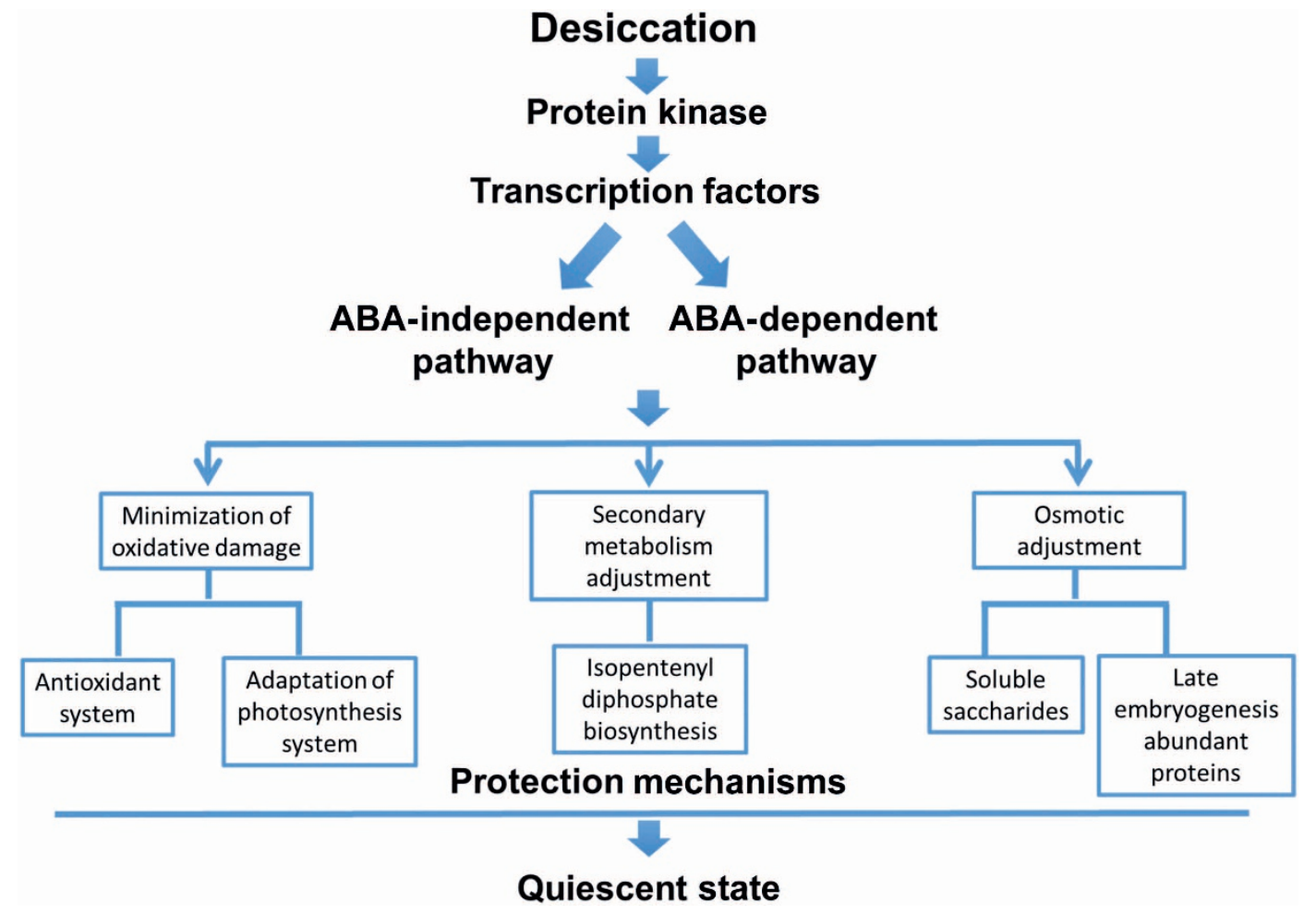

Figure 10. Strategies in the response to desiccation in M. flabellifolia.

and reduced following rehydration, including peroxiredoxins, peroxidases, UDP-glucose 6-dehydrogenases, and 6-phosphogluconate dehydrogenase (Figure 9). In addition, down-regulation of photosynthesis-regulated genes has been observed in other resurrection plants like $C$. plantagineum ${ }^{6}$ and $H$. rhodopensis. ${ }^{20}$ In $M$. flabellifolia, we observed down-regulation of genes encoding subunits of the photosystem reaction center (PsaA/PsaB, PsbP), electron transport chain proteins (ferredoxins, Cytochrome c), and ATP synthase (Supplementary File 11). This response presumably helps the desiccated plant to minimize excitation energy and to reduce ROS production. In striking contrast, several genes encoding lightharvesting antenna system proteins were up-regulated and remained at relatively high expression levels during dehydration and rehydration, including light-harvesting complex proteins (LHCS) of photosystems I and II and early light-inducible proteins (ELIPs) (Figure 9 and Supplementary File 11). For example, the strongest induction in the slightly dehydrated stage was observed for genes encoding chlorophyll $A / B$ binding protein (comp51438_c0_seq1, induced 7438 times) and rubisco activase (comp40977_c0_seq5, induced 5443 times), and in the desiccated stage, it was for a gene encoding ELIP (comp50912_c0_seq1, induced 17809 times). In Arabidopsis, members of the LHC family play roles in the response to ABA. Reducing expression of one $\mathrm{LHC}$ member, $L H C B 6$, decreases drought stress tolerance, ${ }^{75}$ suggesting that up-regulation of LHCs serves as a protective strategy by transducing $A B A$ signaling and modulating ROS homeostasis. Induction of ELIPs was reported to be involved in desiccation stress in resurrection plants like T. ruralis, ${ }^{76}$ C. plantagineum, ${ }^{77}$ and H. rhodopensis. ${ }^{20}$ Functional analysis demonstrated that ELIPs play an important role in protection against chlorophyll-induced photooxidative damage in Arabidopsis. ${ }^{78}$ In $\mathrm{H}$. rhodopensis, a temporary desiccation-induced shift from C3-to-CAM metabolism is enabled by induction of genes encoding phosphoenolpyruvate carboxylase (PEPC). This C3-to-CAM shift may increase water use efficiency and decrease photo-respiratory $\mathrm{H}_{2} \mathrm{O}_{2}$ production. ${ }^{20}$ However, in our analysis, the genes encoding PEPC (comp41025_c0_seq1, comp49907_c0_seq13, comp50447_c1_seq2) in M. flabellifolia decreased in response to desiccation stress (Supplementary File 4), suggesting that different strategies for photosynthetic protection may be employed by M. flabellifolia.

Osmotic responses. During dehydration stress, the initial avoidance of water loss in cells is accomplished by the production of compatible solutes for osmotic adjustment. Several soluble saccharides have been observed to accumulate in desiccated leaves of $M$. flabellifolia, including arbutin, ${ }^{29}$ sucrose, ${ }^{29,31,32}$ trehalose, ${ }^{32}$ and glucopyranosyl- $\beta$-glycerol. ${ }^{31}$ Our data showed that three DTGs encoding sucrose phosphate synthase (SPS, comp50138_c2, comp48008_c0, comp49692_c1) were up-regulated during dehydration (Supplementary File 4). In the resurrection plant S. stapfianus, increases in hexoses and sucrose were associated with increased activity of SPS at the initiation of dehydration, whereas starch content declined to undetectable levels. ${ }^{79}$ The accumulation of SPS in $M$. flabellifolia may redirect the carbon flow from starch to soluble saccharides. Moreover, a DTG encoding UDP-dependent glycosyltransferase (comp46140_c0_seq1, Supplementary File 4) was highly induced under desiccation stress. In Rauvolfia serpentina, UDP-dependent glycosyltransferase is a key enzyme of arbutin biosynthesis. $^{80}$

\section{CONCLUSIONS}

The resurrection plant $M$. flabellifolia has remarkable tolerance to desiccation. Next generation high-throughput sequencing and de novo assembly strategies permitted us to dissect the transcriptome of $M$. flabellifolia during both dehydration and rehydration. Adaptation to desiccation in M. flabellifolia is initiated by the induction of a large number of diverse PKs and TFs. These regulators transduce signal cascades of molecular pathways, including the 
up-regulation of ABA-dependent and independent drought stress pathways and the activation of protective mechanisms for coping with oxidative damage. Antioxidant systems are up-regulated, and the photosynthetic system is modified to reduce ROS generation. Secondary metabolism may participate in desiccation tolerance by the adjustment of isopentenyl diphosphate biosynthesis. The upregulation of genes encoding LEA proteins and sucrose phosphate synthases is also associated with increased tolerance to desiccation (Figure 10).

\section{COMPETING INTERESTS}

The authors declare no conflict of interest.

\section{ACKNOWLEDGMENTS}

We thank Dr. Matthew Opel (University of Connecticut) for providing Myrothamnus flabellifolia plants and Ayla Norris for careful editing of this manuscript. This work was supported by the USDA CRIS project (5306-21000-019-00D), USDA Floriculture Initiative (530613210-001-02S) and Jiangsu Province Natural Science Foundation, China (No. BK20141383).

\section{REFERENCES}

1 Rascio N, La Rocca N. Resurrection plants: the puzzle of surviving extreme vegetative desiccation. Crit Rev Plant Sci 2005; 24: 209-225.

2 Moore JP, Le NT, Brandt WF, Driouich A, Farrant JM. Towards a systems-based understanding of plant desiccation tolerance. Trends Plant Sci 2009; 14: 110-117.

3 Oliver MJ. Desiccation tolerance in vegetative plant cells. Physiol Plant 1996; 97 779-787.

4 Porembski S, Barthlott W. Granitic and gneissic outcrops (inselbergs) as centers of diversity for desiccation-tolerant vascular plants. Plant Ecol 2000; 151: 19-28.

5 Farrant JM, Moore JP. Programming desiccation-tolerance: from plants to seeds to resurrection plants. Curr Opin Plant Biol 2011; 14: 340-345.

6 Rodriguez MCS, Edsgard D, Hussain SS, Alquezar D, Rasmussen M et al. Transcriptomes of the desiccation-tolerant resurrection plant Craterostigma plantagineum. Plant J 2010; 63: 212-228.

7 Bartels D, Salamini F. Desiccation tolerance in the resurrection plant Craterostigma plantagineum. A contribution to the study of drought tolerance at the molecular level. Plant Physiol 2001; 127: 1346-1353.

8 Nakashima K, Yamaguchi-Shinozaki K. Regulons involved in osmotic stressresponsive and cold stress-responsive gene expression in plants. Physiol Plant 2006; 126: 62-71.

9 Shinozaki K, Yamaguchi-Shinozaki K, Seki M. Regulatory network of gene expression in the drought and cold stress responses. Curr Opin Plant Biol 2003; 6: 410-417

10 Leprince O, Buitink J. Desiccation tolerance: From genomics to the field. Plant SCi 2010; 179: 554-564.

11 Weng K, Li Z-Q, Liu R-Q, Wang L, Wang Y-J, Xu Y. Transcriptome of Erysiphe necator infected Vitis pseudoreticulata leaves provides insight into grapevine resistance to powdery mildew. Hort Res 2014; 1: 14049.

12 Zhang S, Chen W, Xin L, Gao Z, Hou Y et al. Genomic variants of genes associated with three horticultural traits in apple revealed by genome re-sequencing. Hort Res 2014; 1: 14045.

13 Gapper NE, Giovannoni JJ, Watkins CB. Understanding development and ripening of fruit crops in an 'omics' era. Hort Res 2014; 1: 14034.

14 Alkio M, Jonas U, Declercq M, Van Nocker S, Knoche M. Transcriptional dynamics of the developing sweet cherry (Prunus avium L.) fruit: sequencing, annotation and expression profiling of exocarp-associated genes. Hort Res 2014; 1: 11.

15 Neale AD, Blomstedt CK, Bronson P, Le TN, Guthridge K et al. The isolation of genes from the resurrection grass Sporobolus stapfianus which are induced during severe drought stress. Plant Cell Environ 2000; 23: 265-277.

16 Oliver MJ. Influence of protoplasmic water loss on the control of protein synthesis in the desiccation-tolerant moss Tortula ruralis: ramifications for a repair-based mechanism of desiccation tolerance. Plant Physiol 1991; 97: 1501-1511.

17 Ingle RA, Schmidt UG, Farrant JM, Thomson JA, Mundree SG. Proteomic analysis of leaf proteins during dehydration of the resurrection plant Xerophyta viscosa. Plant Cell Environ 2007; 30: 435-446.

18 Jiang G, Wang Z, Shang H, Yang W, Hu Z et al. Proteome analysis of leaves from the resurrection plant Boea hygrometrica in response to dehydration and rehydration. Planta 2007; 225: 1405-1420.
19 Rohrig H, Schmidt J, Colby T, Brautigam A, Hufnagel P, Bartels D. Desiccation of the resurrection plant Craterostigma plantagineum induces dynamic changes in protein phosphorylation. Plant Cell Environ 2006; 29: 1606-1617.

20 Gechev TS, Benina M, Obata T, Tohge T, Sujeeth $\mathrm{N}$ et al. Molecular mechanisms of desiccation tolerance in the resurrection glacial relic Haberlea rhodopensis. Cell $\mathrm{Mol}$ Life Sci 2013; 70: 689-709.

21 Moore JP, Lindsey GG, Farrant JM, Brandt WF. An overview of the biology of the desiccation-tolerant resurrection plant Myrothamnus flabellifolia. Ann Bot 2007; 99: 211-217.

22 Moore JP, Nguema-Ona E, Chevalier L, Lindsey GG, Brandt WF et al. Response of the leaf cell wall to desiccation in the resurrection plant Myrothamnus flabellifolius. Plant Physiol 2006; 141: 651-662.

23 Moore JP, Hearshaw M, Ravenscroft N, Lindsey GG, Farrant JM, Brandt WF. Desiccation-induced ultrastructural and biochemical changes in the leaves of the resurrection plant Myrothamnus flabellifolia. Aust J Bot 2007; 55: 482-491.

24 Farrant JM, Cooper K, Kruger LA, Sherwin HW. The effect of drying rate on the survival of three desiccation-tolerant angiosperm species. Ann Bot 1999; 84: 371-379.

25 Schneider H, Manz B, Westhoff M, Mimietz S, Szimtenings M et al. The impact of lipid distribution, composition and mobility on xylem water refilling of the resurrection plant Myrothamnus flabellifolia. New Phytol 2003; 159: 487-505.

26 Moore JP, Farrant JM, Driouich A. A role for pectin-associated arabinans in maintaining the flexibility of the plant cell wall during water deficit stress. Plant Signal Behav 2008; 3: 102-104.

27 Moore JP, Westall KL, Ravenscroft N, Farrant JM, Lindsey GG, Brandt WF. The predominant polyphenol in the leaves of the resurrection plant Myrothamnus flabellifolius, 3,4,5 tri-O-galloylquinic acid, protects membranes against desiccation and free radical-induced oxidation. Biochem J 2005; 385: 301-308.

28 Pizzi A, Cameron FA. Flavonoid tannins-structural wood components for droughtresistance mechanisms of plants. Wood Sci Technol 1986; 20: 119-124.

29 Suau R, Cuevas A, Valpuesta V, Reid MS. Arbutin and sucrose in the leaves of the resurrection plant Myrothamnus flabellifolia. Phytochemistry 1991; 30: 25552556.

30 Moore JP, Farrant JM, Lindsey GG, Brandt WF. The South African and Namibian populations of the resurrection plant Myrothamnus flabellifolius are genetically distinct and display variation in their galloylquinic acid composition. $J$ Chem Ecol 2005; 31: 2823-2834

31 Bianchi G, Gamba A, Limiroli R, Pozzi N, Elster R et al. The unusual sugar composition in leaves of the resurrection plant Myrothamnus flabellifolia. Physiol Plant 1993; 87: 223-226.

32 Drennan PM, Smith MT, Goldsworthy D, Vanstaden J. The occurrence of trehalose in the leaves of the desiccation-tolerant angiosperm Myrothamnus flabellifolius Welw. J Plant Physiol 1993; 142: 493-496.

33 Hopper DW, Ghan R, Cramer GR. A rapid dehydration leaf assay reveals stomatal response differences in grapevine genotypes. Hort Res 2014; 1: 2 .

34 van Nocker S, Gardiner SE. Breeding better cultivars, faster: applications of new technologies for the rapid deployment of superior horticultural tree crops. Hort Res 2014; 1: 14022

35 Grabher MG, Haas BJ, Yassour M, Levin JZ, Thompson DA et al. Full-length transcriptome assembly from RNA-seq data without a reference genome. Nat Biotechnol 2011; 29: 644-652.

36 Chang XX, Donnelly L, Sun D, Rao J, Reid MS, Jiang CZ. A petunia homeodomainleucine zipper protein, PhHD-Zip, plays an important role in flower senescence. PLoS One 2014; 9: e88320

37 Qin Y, Wang M, Tian Y, He W, Han L, Xia G. Over-expression of TaMYB33 encoding a novel wheat MYB transcription factor increases salt and drought tolerance in Arabidopsis. Mol Biol Rep 2012; 39: 7183-7192.

38 Livak KJ, Schmittgen TD. Analysis of relative gene expression data using real-time quantitative PCR and the $2^{-\Delta \Delta C T}$ method. Method 2001; 25: 402-408.

39 Lange BM, Croteau R. Isopentenyl diphosphate biosynthesis via a mevalonateindependent pathway: isopentenyl monophosphate kinase catalyzes the terminal enzymatic step. Proc Natl Acad Sci U S A 1999; 96: 13714-13719.

40 Kirby J, Keasling JD. Biosynthesis of plant isoprenoids: perspectives for microbial engineering. Annu Rev Plant Biol 2009; 60: 335-355.

41 Pulido $\mathrm{P}$, Perello $\mathrm{C}$, Rodriguez-Concepcion M. New insights into plant isoprenoid metabolism. Mol Plant 2012; 5: 964-967.

42 Sherwin HW, Farrant JM. Differences in rehydration of three desiccation-tolerant angiosperm species. Ann Bot 1996; 78: 703-710.

43 Shinozaki K, Yamaguchi-Shinozaki K. Gene networks involved in drought stress response and tolerance. J Exp Bot 2007; 58: 221-227.

44 Yang S, Vanderbeld B, Wan J, Huang Y. Narrowing down the targets: towards successful genetic engineering of drought-tolerant crops. Mol Plant 2010; 3: 469-490. 
45 Abe H, Urao T, Ito T, Seki M, Shinozaki K, Yamaguchi-Shinozaki K. Arabidopsis AtMYC2 (bHLH) and AtMYB2 (MYB) function as transcriptional activators in abscisic acid signaling. Plant Cell 2003; 15: 63-78.

$46 \mathrm{Li} \mathrm{H}$, Sun J, Xu Y, Jiang H, Wu X, Li C. The bHLH-type transcription factor AtAIB positively regulates ABA response in Arabidopsis. Plant Mol Biol 2007; 65: 655-665.

47 Mengiste T, Chen X, Salmeron J, Dietrich R. The Botrytis Susceptible 1 gene encodes an R2R3 MYB transcription factor protein that is required for biotic and abiotic stress responses in Arabidopsis. Plant Cell 2003; 15: 2551-2565.

48 Ulker B, Somssich IE. WRKY transcription factors: from DNA binding towards biological function. Curr Opin Plant Biol 2004; 7: 491-498.

49 Wang M, Vannozzi A, Wang G, Liang YH, Tornielli GB et al. Genome and transcriptome analysis of the grapevine (Vitis vinifera L.) WRKY gene family. Hort Res 2014; 1: 14016.

$50 \mathrm{WuX}$, Shiroto Y, Kishitani S, Ito Y, Toriyama K. Enhanced heat and drought tolerance in transgenic rice seedlings overexpressing OsWRKY11 under the control of HSP101 promoter. Plant Cell Rep 2009; 28: 21-30.

51 Guo AY, Zhu QH, Gu XC, Ge S, Yang J, Luo JC. Genome-wide identification and evolutionary analysis of the plant specific SBP-box transcription factor family. Gene 2008; 418: 1-8.

52 Skirycz A, Claeys H, De Bodt S, Shinoda S, Andriankaja M et al. Pause-and-stop: the effects of osmotic stress on cell proliferation during early leaf development in Arabidopsis and a role for ethylene signaling in cell cycle arrest. Plant Cell 2011; 23: 1876-1888.

53 Xu Y, Gao S, Yang Y, Huang M, Cheng L et al. (2013) Transcriptome sequencing and whole genome expression profiling of chrysanthemum under dehydration stress. BMC Genomics 2013; 14: 662.

54 Dowrick GJ. The chromosomes of Chrysanthemum. Heredity 1953; 7: 59-72.

55 Lehti-Shiu MD, Zou C, Hanada K, Shiu SH. Evolutionary history and stress regulation of plant receptor-like kinase/pelle genes. Plant Physiol 2009; 150: 12-26.

56 Osakabe Y, Yamaguchi-Shinozaki K, Shinozaki K, Tran LS. Sensing the environment key roles of membrane-localized kinases in plant perception and response to abiotic stress. J Exp Bot 2013; 64: 445-458.

57 Shiu SH, Bleecker AB. Plant receptor-like kinase gene family: diversity, function, and signaling. Sci Signal 2001; 1: re22.

58 Kilian J, Whitehead D, Horak J, Wanke D, Weinl S et al. The AtGenExpress global stress expression data set: protocols, evaluation and model data analysis of UV-B light, drought and cold stress responses. Plant J 2007; 50: 347-363.

59 Osakabe Y, Maruyama K, Seki M, Satou M, Shinozaki K, Yamaguchi-Shinozaki K. Leucine-rich repeat receptor-like kinase 1 is a key membrane-bound regulator of abscisic acid early signaling in Arabidopsis. Plant Cell 2005; 17: 1105-1119.

60 Tanaka H, Osakabe Y, Katsura S, Mizuno S, Maruyama K et al. (2012) Abiotic stressinducible receptor-like kinases negatively control ABA signaling in Arabidopsis. Plant J 2012; 70: 599-613.

61 Sinha AK, Jaggi M, Raghuram B, Tuteja N. Mitogen-activated protein kinase signaling in plants under abiotic stress. Plant Signal Behav 2011; 6: 196-203.

62 Mizoguchi T, Irie K, Hirayama T, Hayashida N, Yamaguchi-Shinozaki K et al. A gene encoding a mitogen-activated protein kinase kinase kinase is induced simultaneously with genes for a mitogen-activated protein kinase and an S6 ribosomal protein kinase by touch, cold, and water stress in Arabidopsis thaliana. Proc Natl Acad Sci U S A 1996; 93: 765-769.

63 Ichimura K, Mizoguchi T, Yoshida R, Yuasa T, Shinozaki K. Various abiotic stresses rapidly activate Arabidopsis MAP kinases ATMPK4 and ATMPK6. Plant J 2000; 24: 655-665.

64 Ahlfors R, Macioszek V, Rudd J, Brosché M, Schlichting R et al. Stress hormoneindependent activation and nuclear translocation of mitogen-activated protein kinases in Arabidopsis thaliana during ozone exposure. Plant J 2004; 40: 512-522.
65 Droillard M, Boudsocq M, Barbier-Brygoo H, Lauriere C. Different protein kinase families are activated by osmotic stresses in Arabidopsis thaliana cell suspensions. Involvement of the MAP kinases AtMPK3 and AtMPK6. FEBS Lett 2002; 527: 43-50.

66 Jammes F, Song C, Shin D, Munemasa S, Takeda K et al. MAP kinases MPK9 and MPK12 are preferentially expressed in guard cells and positively regulate ROSmediated ABA signaling. Proc Natl Acad Sci U S A 2009; 106: 20520-20525.

67 Liu Q, Kasuga M, Sakuma Y, Abe H, Miura S et al. Two transcription factors, DREB1 and DREB2, with an EREBP/AP2 DNA binding domain separate two cellular signal transduction pathways in drought- and low-temperature-responsive gene expression, respectively, in Arabidopsis. Plant Cell 1998; 10: 1391-1406.

68 Feuillet C, Schachermayr G, Keller B. Molecular cloning of a new receptor-like kinase gene encoded at the Lr10 disease resistance locus of wheat. Plant J 1997; 11: $45-52$

69 Cheng DW, Armstrong KC, Tinker N, Wight CP, He S et al. Genetic and physical mapping of Lrk10-like receptor kinase sequences in hexaploid oat (Avena sativa L.). Genome 2002; 45: 100-109.

70 Royo J, Gomez E, Balandin M, Muniz LM, Hueros G. ZmLrk-1, a receptor-like kinase induced by fungal infection in germinating seeds. Planta 2006; 223: 1303-1314

71 Furini A, Koncz C, Salamini F, Bartels D. High level transcription of a member of a repeated gene family confers dehydration tolerance to callus tissue of Craterostigma plantagineum. EMBO J 1997; 16: 3599-3608.

72 Schiller $\mathrm{P}$, Heilmeier $\mathrm{H}$, Hartung W. Abscisic acid (ABA) relations in the aquatic resurrection plant Chamaegigas intrepidus under naturally fluctuating environmental conditions. New Phytol 1997; 136: 603-611.

73 Gaff DF, Loveys BR. Abscisic-acid content and effects during dehydration of detached leaves of desiccation tolerant plants. J Exp Bot 1984; 35: 1350-1358.

74 Hoekstra FA, Golovina EA, Buitink J. Mechanisms of plant desiccation tolerance. Trends Plant Sci 2001; 6: 431-438.

75 Xu YH, Liu R, Yan L, Liu ZQ, Jiang SC et al. Light-harvesting chlorophyll a/b-binding proteins are required for stomatal response to abscisic acid in Arabidopsis. J Exp Bo 2012; 63: 1095-1106.

76 Zeng Q, Chen X, Wood AJ. Two early light-inducible protein (ELIP) CDNAs from the resurrection plant Tortula ruralis are differentially expressed in response to desiccation, rehydration, salinity, and high light. J Exp Bot 2002; 53: 1197-1205.

77 Alamillo JM, Bartels D. Effects of desiccation on photosynthesis pigments and the ELIP-like dsp 22 protein complexes in the resurrection plant Craterostigma plantagineum. Plant Sci 2001; 160: 1161-1170.

78 Hutin C, Nussaume L, Moise N, Moya I, Kloppstech K, Havaux M. Early light-induced proteins protect Arabidopsis from photooxidative stress. Proc Natl Acad Sci U S A 2003; 100: 4921-4926.

79 Whittaker A, Martinelli T, Farrant JM, Bochicchio A, Vazzana C. Sucrose phosphate synthase activity and the co-ordination of carbon partitioning during sucrose and amino acid accumulation in desiccation-tolerant leaf material of the $\mathrm{C4}$ resurrection plant Sporobolus stapfianus during dehydration. J Exp Bot 2007; 58 3775-3787.

80 Hefner T, Arend J, Warzecha H, Siems K, Stockigt J. Arbutin synthase, a novel member of the NRD1 $\beta$ glycosyltransferase family, is a unique multifunctional enzyme converting various natural products and xenobiotics. Bioorg Med Chem 2002; 10: 1731-1741.

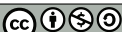

This work is licensed under a Creative Commons AttributionNonCommercial-ShareAlike 4.0 Unported License. The images or other third party material in this article are included in the article's Creative Commons license, unless indicated otherwise in the credit line; if the material is not included under the Creative Commons license, users will need to obtain permission from the license holder to reproduce the material. To view a copy of this license, visit http://creativecommons.org/ licenses/by-nc-sa/4.0/

Supplementary information accompanies this article and can be found on the Horticulture Research's website (http://www.nature.com/hortres). 